\title{
Lymphocytes are dispensable for glomerulonephritis but required for renal interstitial fibrosis in matrix defect-induced Alport renal disease
}

\author{
Valerie S LeBleu ${ }^{1,2}$, Hikaru Sugimoto ${ }^{1}$, Caroline A Miller ${ }^{3}$, Vincent H Gattone $\|^{3}$ and Raghu Kalluri ${ }^{1,2,4}$
}

One current theory for the emergence of glomerular nephritis implicates Th1-type cellular responses associated with delayed-type hypersensitivity, involving $T$ cells and macrophages. Using a mouse model for progressive glomerulonephritis, we investigate the role of B and T cells in the pathogenesis of glomerular inflammation. Deletion of $\alpha 3$ chain of type IV collagen in mice ( $\alpha 3(\mathrm{IV})$ collagen null mice) results in GBM defects, glomerulonephritis and tubulointerstitial inflammation, fibrosis and significant immune infiltration including activated B- and T-lymphocytes. To evaluate the contribution of lymphocytes to the pathogenesis of glomerulonephritis and renal fibrosis, we generated mice that are deficient in both the $\alpha 3$ (IV) collagen and Rag-1 ( $\alpha 3 /$ Rag-1 DKO). Lymphocyte deficiency significantly reduces fibrosis in the renal interstitium, but ultrastructural GBM defects persist. Interestingly, glomerulonephritis in the double null mice persists at a similar level with comparable proteinuria. Here we demonstrate that despite the presence of B-cell and T-cells in the inflamed glomeruli, their deletion does not impede the emergence of glomerulonephritis but has a negative impact on the progression of renal interstitial fibrosis.

Laboratory Investigation (2008) 88, 284-292; doi:10.1038/labinvest.3700715; published online 7 January 2008

KEYWORDS: crescentic glomerulonephritis; $\alpha 3(\mathrm{IV})$ collagen KO; Rag-1 KO; renal fibrosis; Alport

Glomerulonephritis and renal fibrosis is an indicator of the severity of kidney disease in human kidney biopsies and is associated with poor prognosis. ${ }^{1-4}$ Unless spontaneously resolved, some form of glomerulonephritis progresses to more advanced fibro-cellular and fibrous crescents, irreversibly compressing and scaring the glomeruli and disabling its filtration property. A better understanding of the pathogenesis of glomerulonephritis may lead to the development of new therapeutic strategies. Nephrotoxic nephritis rodent models, in which the animals are given heterologous antisera to the glomerular basement membrane (GBM), are most commonly studied for the emergence of glomerulonephritis. In these models, the mechanisms for glomerulonephritis implicates a $\mathrm{CD} 3+\mathrm{T}$ cell-driven Th1-type responses with delayed-type hypersensitivity. ${ }^{5-13}$ In contrast, Ophascharoensuk et $a l^{14}$ report that in their model of murine nephritis, proliferating glomerular epithelial cells are the main cellular components of the crescent, with early crescents devoid of macrophages and $\mathrm{T}$ cells. Furthermore, a growing body of evidence has suggested that parietal epithelial cells are involved in crescent formation, with transdifferentiation of parietal epithelial cells and acquisition of new epitopes, shared by macrophages. ${ }^{15-23}$ These results indicate that origins of glomerulonephritis still remain ambiguous, and mechanisms may differ according to the underlying renal disease.

In this study, we investigate the roles of $\mathrm{B}$ and $\mathrm{T}$ cells in the pathogenesis of glomerulonephritis in the type IV collagen $\alpha 3$ chain null mice. Deletion of the type IV collagen $\alpha 3$ chain ( $\alpha 3 \mathrm{KO}$ mice) impairs the obligate type IV collagen $\alpha 3 / \alpha 4 / \alpha 5$ protomer formation in the GBM. ${ }^{24}$ The $\alpha 3$ (IV) collagen null mouse is one of the animal models used to study the pathophysiology of Alport Syndrome. ${ }^{25,26}$ These mice are deficient in producing type IV collagen $\alpha 3$ chain and develops progressive glomerulonephritis, tubulo-interstitial disease, and thinning and focal multilaminated thickening of the GBM. ${ }^{24,26,27}$ The background strain of mouse strongly affects the disease progression and survival of the $\alpha 3$ (IV)

\footnotetext{
${ }^{1}$ Division of Matrix Biology, Beth Israel Deaconess Medical Center and Harvard Medical School, Boston, MA, USA; ${ }^{2}$ Department of Biological Chemistry and Molecular Pharmacology, Harvard Medical School, Boston, MA, USA; ${ }^{3}$ Department of Anatomy and Cell Biology, Indiana University School of Medicine, Indianapolis, IN, USA and ${ }^{4}$ Harvard-MIT Division of Health Sciences and Technology, Boston, MA, USA

Correspondence: Dr R Kalluri, PhD, Division of Matrix Biology, Beth Israel Deaconess Medical Center, Harvard Medical School, 330, Brookline Avenue (DANA 514), Boston, MA 02115, USA. E-mail: rkalluri@BIDMC.Harvard.edu
} 
collagen null mice. This modifier effect implies that GBM make up and the immune response towards the damaged GBM is likely influenced by inherent genotypic differences. $^{28,29}$ The $\alpha 3(\mathrm{IV})$ collagen null mice on $\mathrm{C} 57 \mathrm{Bl} / 6 \mathrm{~J}$ background exhibit detectable proteinuria starting at 12 weeks of age and die of end stage renal failure between 22-27 weeks of age. ${ }^{24}$

It is proposed that a robust lymphocytic infiltration in the damaged kidneys is triggered by the GBM structural defects and miscommunications resulting from aberrant cell-matrix interaction in the glomeruli. It is assumed that such infiltration of B-cells and T-cells contribute significantly to the emergence of glomerulonephritis and tubulo-interstitial nephritis leading to tissue fibrosis. To address the specific role of the T- and B-lymphocytes in this process, we crossed the a3(IV) collagen null mice with the Rag-1 null mice and analyzed the disease progression in these double null mice ( $\alpha 3 /$ Rag-1 DKO mice). The deletion of the Rag-1 gene results in a blockade of lymphocyte differentiation by disabling $\mathrm{V}(\mathrm{D}) \mathrm{J}$ recombination. ${ }^{30}$ While mature $\mathrm{B}$ - and T-cells are absent in the Rag-1 null mice, NK cells, neutrophils and macrophages are present in these mice.

Our results demonstrate that B- and T-lymphocytes are not required for the glomerulonephritis but are essential components of tubulointerstitial inflammation and fibrosis. Collectively, our study highlights that the pathogenesis of glomerulonephritis does not involve B and T-lymphocytes in the $\alpha 3$ (IV) collagen null mice.

\section{MATERIALS AND METHODS Mice}

The generation and disease progression in the $\alpha 3 \mathrm{KO}$ mice was described previously. ${ }^{24,26}$ The mice were backcrossed (10 generations) into the C57BL/6J background. The Rag-1 KO (C57BL6/J background) mice were purchased from the Jackson Laboratory. All animals with the Rag-1 gene deletion were housed in autoclaved cages with irradiated murine chow and autoclaved water. The $\alpha 3 \mathrm{KO}$ mice were housed under standard conditions. All animals were housed in the Beth Israel Deaconess Medical Center animal facility. All animal studies were reviewed and approved by the Animal Care and Use Committee of the Beth Israel Deaconess Medical Center.

\section{Urine Albumin/Creatinine}

Urine samples were collected at 8,12 and 22 weeks of age and kept frozen prior to analyses. Creatinine concentration was measured using the colorimetric assay Quantichrome (DICT500) from BioAssays (Hayward, CA, USA) according to the manufacturer's directions. Albumin concentrations were measured using the Mouse Albuminuria ELISA (Bethyl laboratory, Montgomery, TX, USA) according to the manufacturer's direction.

\section{Blood Creatinine}

Blood samples $(100 \mu \mathrm{l})$ were collected at 8,12 and 22 weeks of age. Plasma was separated from cellular components by centrifugation ( $5 \mathrm{~min}, 6000$ r.p.m., RT) and stored frozen prior to analyses. Plasma creatinine was measured using HPLC analysis (Dr Steve Dunn, Thomas Jefferson University, Division of Nephrology Rodent Core Laboratories, Philadelphia, PA, USA).

\section{Kidney Tissue Analyses}

Mice were euthanized using $\mathrm{CO}_{2}$ at 8,12 , and 22 weeks of age and kidneys harvested. The kidneys were dissected and fixed accordingly.

\section{Immunocytochemistry}

Sagittal sections were embedded in OCT compound and snap frozen in liquid nitrogen. Thin frozen sections $(0.6 \mu \mathrm{m})$ were fixed in ice-cold acetone $(20 \mathrm{~min})$ and immunostained against podocin, staining the podocyte (dilution 1:200, a gift from Dr Peter Mundel, Mount Sinai School of Medicine, New York, NY, USA), CD3 (dilution 1:200, eBioscience, 14-0031) and CD19 (dilution 1:250, Serotec MCA1439). TRITC- and FITC-conjugated secondary antibodies (Jackson Immunoresearch) were used at a 1:200 dilution. The slides were mounted with Vectashield Mounting Medium with DAPI (Vectashield; H1200) and glass coverslip, and analyzed using the Axioskop 2 fluorescent microscope, AxioCam HRC camera and the Axiovision 4.3 software.

\section{Light Microscopy}

Kidneys were also fixed in formalin and paraffin sections used for Haematoxylin and Eosin, Periodic Acid Schiff and Masson's Trichrome staining under standard conditions (Histology Core Facility, Beth Israel Deaconess Medical Center, Boston, MA, USA). Morphometric analyses for the histological assessment of renal injury, here glomerular sclerosis and interstitial fibrosis, were performed as described previously. ${ }^{29}$ For the analysis of interstitial fibrosis, Masson's Trichrome-stained kidney sections where observed using a counting grid at $\times 400$ magnification. The number of grid intersections, which coincide with interstitial fibrosis was expressed as a percentage of the total number of grid intersections. Five random views were counted per slide and five slides were observed per experimental group. For the analysis of glomerular sclerosis and crescentic glomeruli, 100 glomeruli were counted per slide and five slides were counted per experimental group. The number of normal glomeruli was expressed as a percentage of the total number of glomeruli counted for glomerular sclerosis and the number of crescentic glomeruli was expressed as a percentage of total glomeruli. Glomerular crescent cellularity was evaluated from 50 glomeruli in five mice per experimental group. The number of cells (nuclei) in the crescent of 10 glomeruli in five mice per experimental group was compared. 


\section{Electron Microscopy}

For transmission electron microscopy, $1-\mathrm{mm}^{3}$ kidney sections were fixed overnight (in $2 \%$ glutaraldehyde in $0.1 \mathrm{M}$ cacodylic acid, $\mathrm{pH}$ 7.4). Tissue specimens were post fixed with $1 \%$ osmium tetroxide, dehydrated and embedded in Embed 812 (Electron Microscopy Sciences, Hatfield, PA, USA). The blocks were sectioned at $75 \mathrm{~nm}$ on a Leica UCT ultramicrotome (Bannockburn, IL, USA) using a Diatome diamond knife (Electron Microscopy Sciences, Hatfield, PA, USA). Images were taken on a Technia G12 Biotwin TEM (FEI, Hillsboro, OR, USA) equipped with an AMT CCD camera (Advanced Microscopy Techniques, Danvers, MA, USA). All processing, sectioning and scope work was done in the Electron Microscopy Center, Department of Anatomy and Cell Biology, at the Indiana University School of Medicine.

\section{Statistical Analyses}

Standard errors were calculated and $t$-test and ANOVA were used to determine statistical differences. A level of $P<0.05$ was considered statistically significant.

\section{RESULTS}

At 8 weeks of age, the $\alpha 3 \mathrm{KO}$ mice exhibit nearly normal renal function and histology. ${ }^{26}$ Twelve-week-old $\alpha 3 \mathrm{KO}$ mice present with detectable proteinuria and mild-to-moderate glomerulonephritis and interstitial fibrosis $(20 \%$ decrease in the number of normal glomeruli). By 22 weeks of age, the mice develop massive proteinuria, severe crescentic glomerulonephritis, glomerulosclerosis, interstitial fibrosis, and ultrastructural defects in the GBM.

\section{Lack of B- and T-lymphocyte Infiltration in the Injured Glomeruli of the Mice}

To confirm a loss of mature B- and T-cell infiltration in the kidneys of 22-week-old $\alpha 3 /$ Rag-1 DKO animals, kidney sections from these mice, the $\alpha 3 \mathrm{KO}$, and the Rag- $1 \mathrm{KO}$ litter mates were labeled for mature $\mathrm{B}(\mathrm{CD} 19)$ and mature $\mathrm{T}$ (CD3) lymphocyte markers. The CD3- or CD19-positive cells were not detected in the Rag- $1 \mathrm{KO}$ and the $\alpha 3 /$ Rag- 1 DKO mice, whereas significant number of T-cells and B-cells were observed in the $\alpha 3 \mathrm{KO}$ animals (Figure 1). T-cells and B-cells were mainly detected in the renal interstitium. Injured glomeruli in the $\alpha 3 \mathrm{KO}$ mice exhibit very few cells that are $\mathrm{CD}^{+}$but none that are $\mathrm{CD} 19^{+} . \mathrm{CD}^{+}$and $\mathrm{CD} 19^{+}$cells are not detected in the inflamed glomeruli of the $\alpha 3 /$ Rag- 1 DKO mice.

\section{Significant Difference is Detected in the Renal Histology Between the $\alpha 3 \mathrm{KO}$ and $\alpha 3 /$ Rag-1 DKO Mice}

Light microscopy analysis Haematoxylin and Eosin (H\&E), Periodic Acid Schiff (PAS) and Masson's Trichrome staining (MTS) was performed using the kidneys from the $\alpha 3 \mathrm{KO}$ and $\alpha 3 /$ Rag-1 DKO mice (Figure 2a). Haematoxylin and Eosin labeling of the renal cortex reveals diffuse glomerular and tubular damage, with shrunken and sclerotic glomeruli and tubular casts (Figure 2a, middle panel). Despite mature lymphocyte ablation in the $\alpha 3 /$ Rag- 1 DKO mice, leukocyte infiltration (likely macrophages, neutrophils, and NK cells) persists in the interstitium of these mice. Periodic Acid Schiff labeling of glomeruli reveals formation of a fibrous cap with epithelial and mesangial hypercellularity in both the $\alpha 3 \mathrm{KO}$ and $\alpha 3 /$ Rag- $1 \mathrm{DKO}$, in contrast to the glomeruli of the Rag-1 $\mathrm{KO}$ animals (Figure 2a, lower panel). The $\alpha 3 \mathrm{KO}$ and $\alpha 3 /$ Rag-1 DKO animals exhibit tubular atrophy and fibrotic scaring, as determined using Masson's Trichrome labeling of the cortical region of the kidney (Figure 2a, upper panel). Morphometric analysis reveals a significant difference in the glomerulosclerosis between the $\alpha 3 \mathrm{KO}$ and $\alpha 3 / \mathrm{Rag}-1 \mathrm{DKO}$ mice (Figure $2 \mathrm{~b}$ ). Tubular atrophy and interstitial fibrosis is significantly greater in the $\alpha 3 \mathrm{KO}$ mice when compared to the $\alpha 3 /$ Rag-1 DKO mice (Figure 2b, lower panel). Morphometric evaluation of kidney sections from 22 -week-old $\alpha 3 \mathrm{KO}$ mice show $\sim 70 \%$ tubular atrophy and $\sim 12 \%$ interstitial fibrosis whereas $\alpha 3 /$ Rag-1 DKO mice show a significant reduction in tubular damage, with $\sim 40 \%$ tubular atrophy and $\sim 5 \%$ interstitial fibrosis. The Rag-1 KO littermates exhibit normal kidney histology. The number of crescentic glomeruli is slightly lower in the $\alpha 3 /$ Rag-1 DKO mice $(\sim 15 \%)$ when compared to the $\alpha 3 \mathrm{KO}$ mice $(\sim 22 \%)$. Cellularity in the crescent is greater in the $\alpha 3 \mathrm{KO}$ mice $(\sim 40 \%)$ when compared to the $\alpha 3 /$ Rag-1 DKO ( $\sim 28 \%$ ) (Figure $2 \mathrm{~b}$, lower panel).

\section{Renal Function in the $\alpha 3 K O$ and $\alpha 3 /$ Rag-1 DKO Mice}

The ratio of urine albumin to urine creatinine was used as a measure of proteinuria and with an increased ratio indicating an increase in glomerular vascular leak via the glomerular pathology. Increased urine protein was observed in the $\alpha 3 \mathrm{KO}$ and $\alpha 3 /$ Rag- 1 DKO mice at 12 and 22 weeks of age, while the Rag-1 KO mice exhibit normal range of urine protein (Figure 3a). Serum creatinine levels in the $\alpha 3 \mathrm{KO}$ and $\alpha 3 / \mathrm{Rag}-1 \mathrm{DKO}$ mice increase as the disease progresses, while the littermate Rag-1 KO animals show normal range of serum creatinine, comparable to wild type mice $(0-0.1 \mathrm{mg} / \mathrm{dl}$, Figure $3 \mathrm{~b})$. Serum creatinine concentration at 22 weeks of age is significantly greater in the $\alpha 3 \mathrm{KO}$ mice $(0.45 \mathrm{mg} / \mathrm{dl})$, when compared to the $\alpha 3 / \mathrm{Rag}-1$ DKO mice $(0.2 \mathrm{mg} / \mathrm{dl})$. This suggests a direct improvement in the interstitial disease of $\alpha 3 \mathrm{KO}$ mice when lymphocytes are deleted.

\section{Ultrastructural GBM Defects in the $\alpha 3 K O$ and $\alpha 3 /$ Rag-1 DKO Mice}

Twelve- and 22-week-old mice from each group were sacrificed and their kidneys harvested and prepared for transmission electron microscope (TEM) analysis (Figure 4). The Rag-1 KO animal presents with a continuous, homogeneous electron dense GBM, similar to the wild-type animals (Figure 4). The GBM in the $\alpha 3 \mathrm{KO}$ and $\alpha 3 /$ Rag- 1 DKO mice reveal thinning, focal multilaminated thickening, 


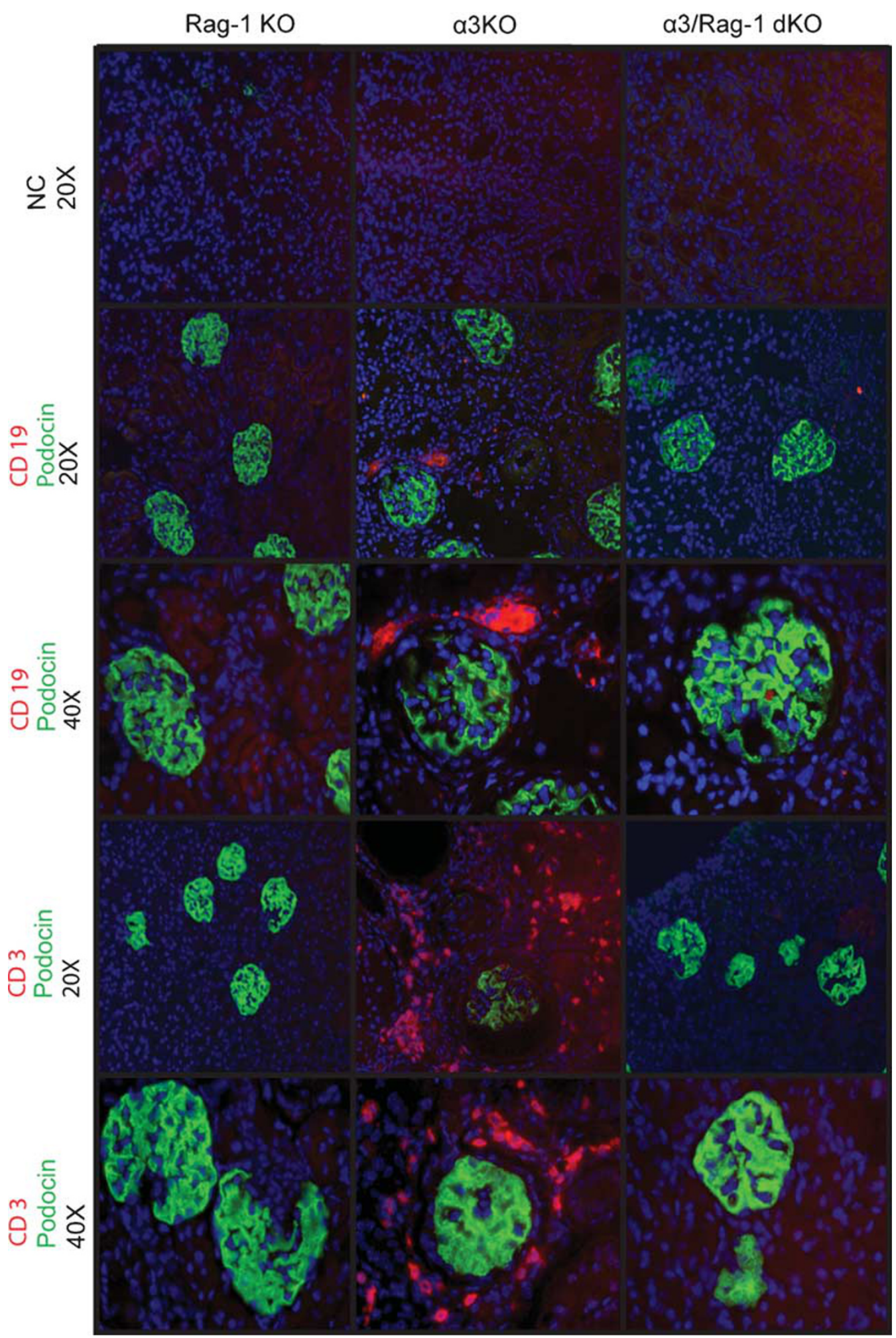

Figure 1 Immunocytochemistry labeling for mature B-cells and T-cells: antibodies were used against CD19 and CD3, cell surface markers were used in the kidney tissue sections of 22-week-old Rag-1 KO, $\alpha 3 \mathrm{KO}$, and $\alpha 3 /$ Rag-1 DKO mice. CD3 and CD19 positive cells are shown in red; podocin staining of podocytes in the glomeruli is shown in green.

splitting and basket weave appearance of the GBM with podocyte foot processes effacement, characteristic of renal disease associated with Alport syndrome (Figure 4). These results suggest that recruitment of lymphocytes within the injured glomeruli do not appear to impact the accumulation of structural defects in the GBM. 
a

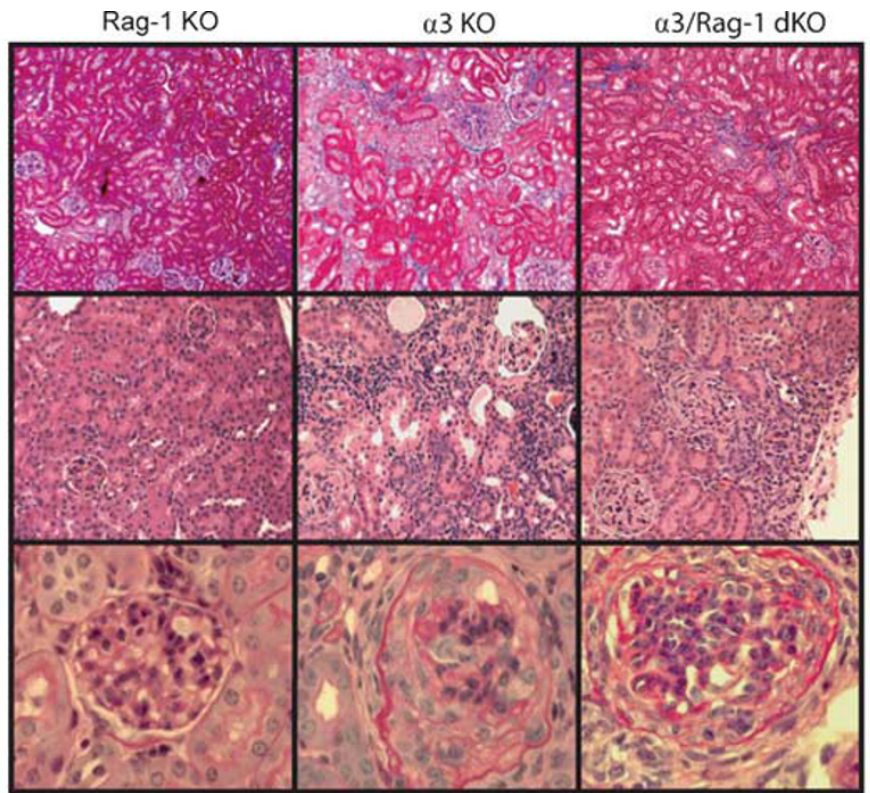

b

PAS, 63X

MTS, 10X

H\&E, 20X

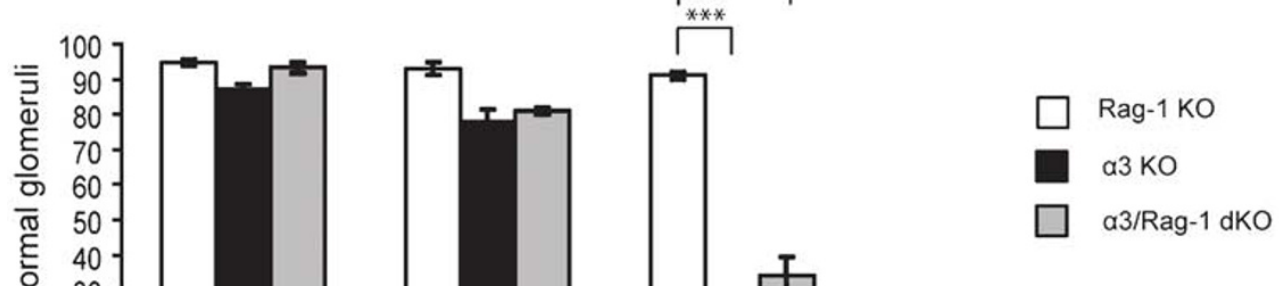

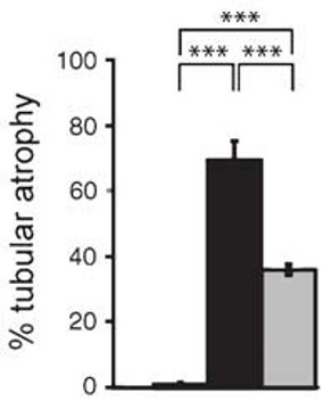

22 weeks

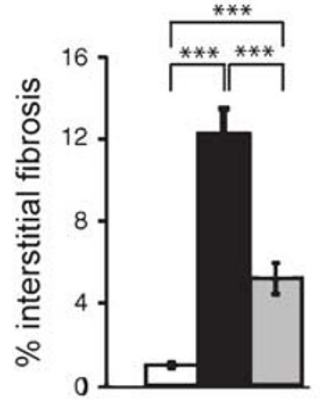

22 weeks

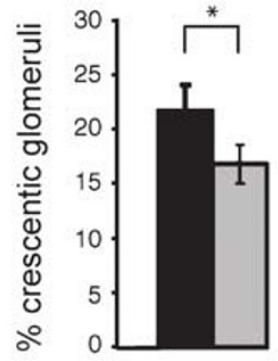

22 weeks

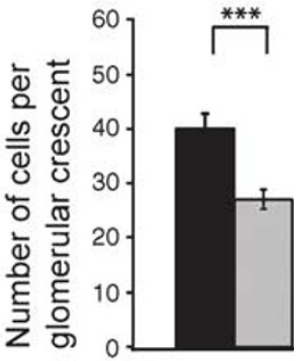

22 weeks

Figure 2 Light microscopy imaging and morphometric analyses. (a) Haematoxylin and Eosin (H\&E), Periodic Acid Schiff (PAS) and Masson's Trichrome staining (MTS) of kidneys from 22-week-old Rag-1 KO, $\alpha 3 \mathrm{KO}$, and $\alpha 3 /$ Rag-1 DKO mice. Respective magnifications are indicated on the figure.

(b) Morphometry of glomerular sclerosis (expressed as \% of normal glomeruli) of 8,12 and 22-week-old Rag-1 KO $(N=5), \alpha 3 \mathrm{KO}(N=5)$, and $\alpha 3 /$ Rag-1 DKO mice $(N=5)$, percent tubular atrophy, interstitial fibrosis, crescentic glomeruli and crescent cellularity of 22-week-old Rag-1 KO $(N=5)$, $\alpha 3 \mathrm{KO}(N=5)$, and $\alpha 3 /$ Rag-1 DKO mice $(N=5) .{ }^{*} P<0.05,{ }^{* * *} P<0.001$ (ANOVA; $t$-test for $\%$ crescentic glomeruli and $\%$ number of cells per glomerular crescent).

\section{DISCUSSION}

The present study reveals that deficiency in lymphocytic infiltration does not prevent glomerulonephritis in the $\alpha 3 \mathrm{KO}$ mice. Our results demonstrate that lymphocytes are not required for the emergence of glomerulonephritis, but may influence the rate of glomerular disease. Based on unbiased statistical analyses of renal function tests and morphometric measurements of renal histology, glomerulonephritis, and 


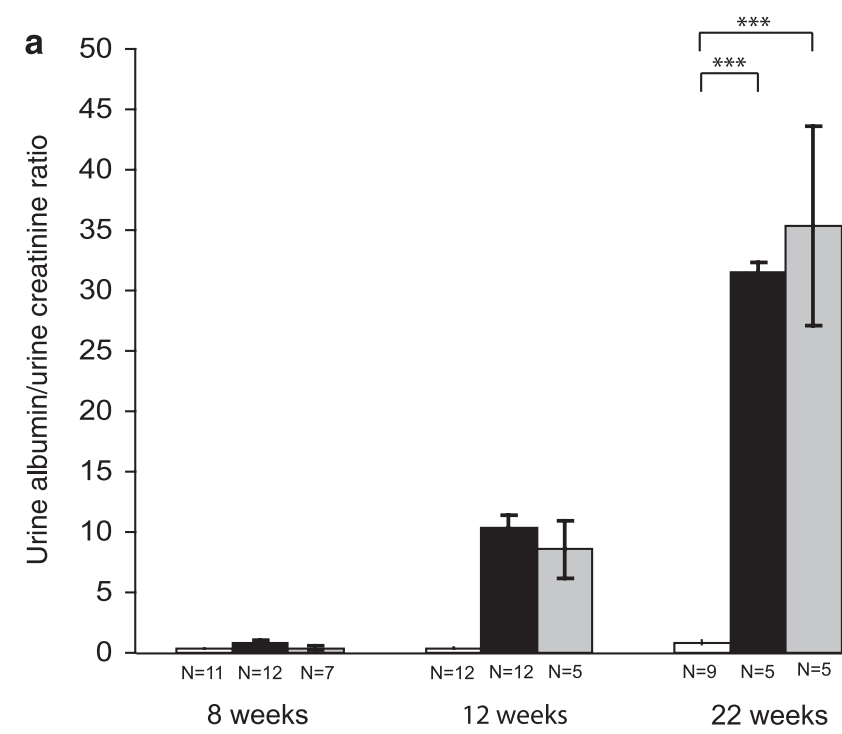

Rag-1 KO

a3 KO

$\alpha 3 /$ Rag-1 dKO

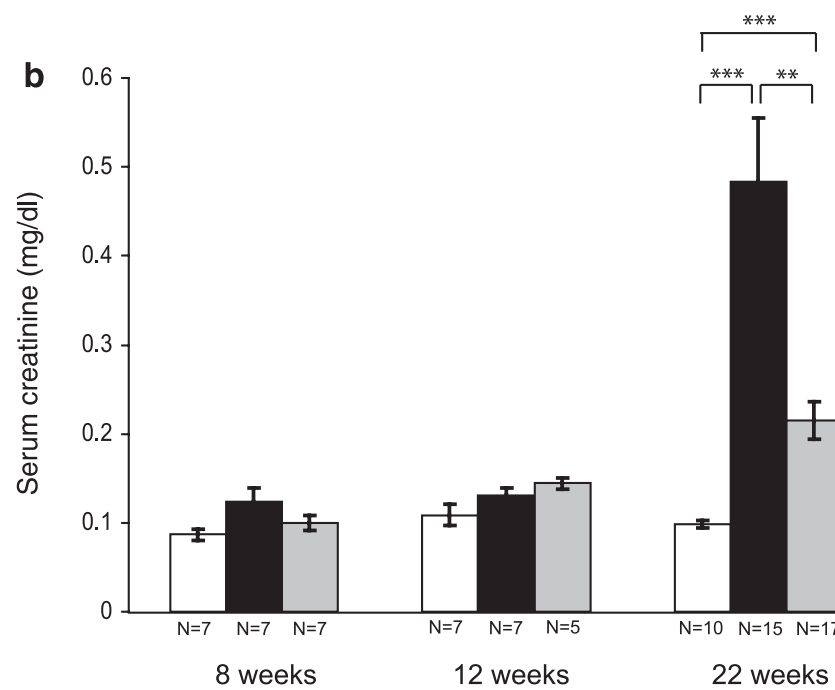

Rag-1 KO

a3 KO

a3/Rag-1 dKO

Figure 3 Glomerular functions. Rag- $1 \mathrm{KO}, \alpha 3 \mathrm{KO}$, and $\alpha 3 /$ Rag-1 DKO mice at 8, 12, and 22 weeks of age were analyzed. Urine albumin/urine creatinine ratio (a) and serum creatinine concentration in $\mathrm{mg} / \mathrm{dl}$ (b) indicate normal glomerular function for the Rag-1 KO animals over time, which was significantly different than for the $\alpha 3 \mathrm{KO}$ and $\alpha 3 /$ Rag-1 DKO animals, showing similar progression in loss of glomerular filtration properties. There were no significant differences in urine albumin/urine creatinine ratio between the $\alpha 3 \mathrm{KO}$ and $\alpha 3 /$ Rag-1 DKO groups. A significant difference in serum creatinine was found between the two groups at 22 weeks of age. $N=$ number of animals per group, ${ }^{* *} P<0.01,{ }^{* * *}<<0.001$ (ANOVA).

proteinuria was determined to persist at a similar level in both $\alpha 3 /$ Rag- 1 DKO and $\alpha 3 \mathrm{KO}$ experimental groups, whereas depletion of active lymphocytes in the DKO animal reduced the injury in the renal interstitium. While the initial insult in the $\alpha 3 \mathrm{KO}$ mice is targeted deletion of a GBM collagen to induce a structural defect, the ensuing injury involves massive influx of immune infiltrate. Our study shows that in the $\alpha 3 \mathrm{KO}$ glomeruli, lymphocytes are not detected, but the renal interstitium contains many $\mathrm{CD}^{+}$and $\mathrm{CD} 19^{+}$ lymphocytes. The GBM structural defect likely results in abnormal endothelium-GBM-podocyte communications. The loss of podocyte attachment to the defective GBM may result in de-differentiation and subsequent proliferation of the visceral epithelium, in addition to spillage of inflammatory mediators into the Bowman's space. Proliferation of the visceral and parietal epithelium could contribute to crescent formation in the $\alpha 3 \mathrm{KO}$ mice. Once the glomerular disease initiates, it progressively leads to glomerulonephritis. ${ }^{16,31}$ Eventually, the glomerular damage spreads to the renal interstitium with tubular damage, atrophy, and fibrosis. Proteinuria is first detected in association with glomerular disease, subsequently serum creatinine levels go up to coincide with renal tubular damage and interstitial fibrosis.

Glomerulonephritis develops despite the ablation of lymphocytes. These results strongly argue that mature T-cells and B-cells are not required for the glomerular disease in the $\alpha 3 \mathrm{KO}$ mice and that adaptive immunity may not play a 

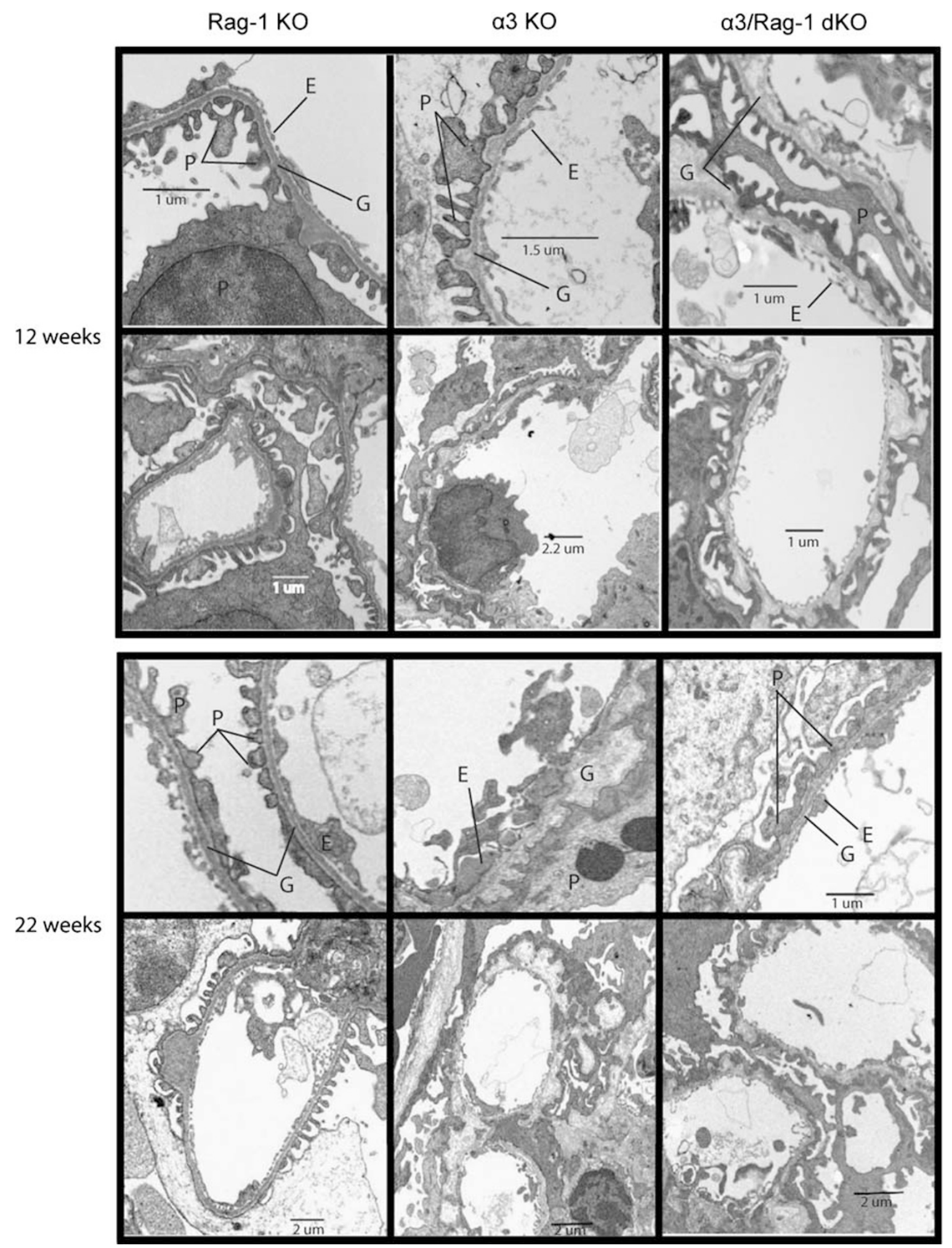

Figure 4 Ultrastructural analyses of the Glomerular Basement Membrane. Transmission Electron Microscopy of 12- and 22-week-old Rag-1 KO, $\alpha 3 \mathrm{KO}$, and «3/Rag-1 DKO mice. P: podocyte foot processes, E: fenestrated endothelium, G: GBM.

role in the emergence of glomerulonephritis, but influences the rate of crescent evolution. While the number of glomerular crescents and inflammation is slightly lower in the $\alpha 3 /$ Rag-1 DKO mice, glomerular function as evidenced by proteinuria, is unaltered in these mice when compared to the $\alpha 3 \mathrm{KO}$ mice. 
Specific autoimmunity towards glomerular antigens in the nephrotoxic nephritis mouse models indicate a possible role for T-cells in glomerulonephritis in this setting. ${ }^{5-9,11-13}$ In contrast, our study demonstrates that T-cell depletion in our mouse model of progressive glomerular nephropathy does not impact the emergence of glomerulonephritis. The cellular composition of glomerular crescents in the $\alpha 3 \mathrm{KO}$ mouse model does not appear to contain $\mathrm{CD}^{+}$and $\mathrm{CD} 19^{+}$ lymphocytes, but is rather composed of proliferating visceral and parietal glomerular epithelial cells, and possibly macrophages, although their identification in glomerular crescents is challenging. ${ }^{15-17,20-22}$

Overall, deletion of B-cell and T-cells in $\alpha 3 \mathrm{KO}$ mice does not result in a protection from glomerular disease and loss of renal function. Proteinuria remains the same in the $\alpha 3 \mathrm{KO}$ mice and the $\alpha 3 / \mathrm{Rag}-1$ DKO mice. Interestingly, ultrastructural analysis by electron microscopy does not reveal any significant difference between the $\alpha 3 \mathrm{KO}$ mice and the $\alpha 3 /$ rag-1 DKO mice. In contrast, absence of lymphocytes leads to significant protection from tubulo-interstitial inflammation. Despite similar leukocyte (macrophages, NK cells, and neutrophils) infiltration in the renal interstitium of both $\alpha 3 \mathrm{KO}$ mice and the $\alpha 3 / \mathrm{rag}-1$ DKO mice, absence of mature $\mathrm{B}$ and $\mathrm{T}$ lymphocytes significantly reduced the injury to the renal interstitium. Nephrogenic T-cell response and T-cell infiltration in interstitial nephritis initiate, amplify, and perpetuate local inflammatory response which contributes to tubular atrophy and interstitial fibrosis. ${ }^{34,36-38}$ However, the emergence of tubular interstitial fibrosis in our $\alpha 3 /$ Rag- 1 DKO mice, as suggested by Ninichuk et al, ${ }^{32}$ could result from macrophage recruitment and activation via MCP-1 expression. ${ }^{33-35}$ Lower serum creatinine levels in the double null mice indicate that ablating active lymphocytes decreases the injury to the renal interstitium. Tubular damage and atrophy is significantly attenuated, resulting in reduced level of fibrosis. This report provides compelling evidence for the role of adaptive immunity in mediating tubular damage and facilitating fibrosis with insignificant impact on the glomerular disease and function.

\section{ACKNOWLEDGEMENT}

This study was partially funded by a research grant from Emerald Foundation, and NIH research grants DK 55001 (RK) and DK62987 (RK) and research funds from the Beth Israel Deaconess Medical Center for the Division of Matrix Biology.

1. Atkins RC, Nikolic-Paterson DJ, Song Q, et al. Modulators of crescentic glomerulonephritis. J Am Soc Nephrol 1996;7:2271-2278.

2. Mathieson PW. The ins and outs of glomerular crescent formation. Clin Exp Immunol 1997;110:155-157.

3. Morrin PA, Hinglais N, Nabarra B, et al. Rapidly progressive glomerulonephritis. A clinical and pathologic study. Am J Med 1978;65:446-460.

4. Whitworth JA, Morel-Maroger L, Mignon F, et al. The significance of extracapillary proliferation. Clinicopathological review of 60 patients. Nephron 1976;16:1-19.
5. Connolly K, Roubinian JR, Wofsy D. Development of murine lupus in CD4-depleted NZB/NZW mice. Sustained inhibition of residual CD4+ T cells is required to suppress autoimmunity. J Immunol 1992;149:3083-3088.

6. Huang XR, Holdsworth SR, Tipping PG. Evidence for delayed-type hypersensitivity mechanisms in glomerular crescent formation. Kidney Int 1994;46:69-78.

7. Huang XR, Tipping PG, Apostolopoulos J, et al. Mechanisms of T cellinduced glomerular injury in anti-glomerular basement membrane (GBM) glomerulonephritis in rats. Clin Exp Immunol 1997;109:134-142.

8. Jabs DA, Burek CL, Hu Q, et al. Anti-CD4 monoclonal antibody therapy suppresses autoimmune disease in MRL/Mp-Ipr/lpr mice. Cell Immunol 1992;141:496-507.

9. Kalluri $\mathrm{R}$, Danoff $T M$, Okada $\mathrm{H}$, et al. Susceptibility to anti-glomerular basement membrane disease and Good pasture syndrome is linked to MHC class II genes and the emergence of T cell-mediated immunity in mice. J Clin Invest 1997;100:2263-2275.

10. Kitching AR, Holdsworth SR, Tipping PG. Crescentic glomerulonephritis-a manifestation of a nephritogenic Th1 response? Histol Histopathol 2000;15:993-1003.

11. Li S, Holdsworth SR, Tipping PG. Antibody independent crescentic glomerulonephritis in mu chain deficient mice. Kidney Int 1997;51:672-678.

12. Tipping PG, Holdsworth SR. T cells in crescentic glomerulonephritis. J Am Soc Nephrol 2006;17:1253-1263.

13. Tipping PG, Huang XR, Qi M, et al. Crescentic glomerulonephritis in CD4- and CD8-deficient mice. Requirement for CD4 but not CD8 cells. Am J Pathol 1998;152:1541-1548.

14. Ophascharoensuk V, Pippin JW, Gordon KL, et al. Role of intrinsic renal cells vs infiltrating cells in glomerular crescent formation. Kidney Int 1998;54:416-425.

15. Bariety J, Bruneval P, Hill G, et al. Post transplantation relapse of FSGS is characterized by glomerular epithelial cell transdifferentiation. J Am Soc Nephrol 2001;12:261-274.

16. Bariety J, Bruneval $P$, Meyrier A, et al. Podocyte involvement in human immune crescentic glomerulonephritis. Kidney Int 2005;68:1109-1119.

17. Bariety J, Nochy D, Mandet $C$, et al. Podocytes undergo phenotypic changes and express macrophagic-associated markers in idiopathic collapsing glomerulopathy. Kidney Int 1998;53:918-925.

18. Kihara I, Yaoita E, Kawasaki K, et al. Origin of hyperplastic epithelial cells in idiopathic collapsing glomerulopathy. Histopathology 1999;34:537-547.

19. $\mathrm{Ng} \mathrm{YY,} \mathrm{Fan} \mathrm{JM,} \mathrm{Mu} \mathrm{W,} \mathrm{et} \mathrm{al.} \mathrm{Glomerular} \mathrm{epithelial-myofibroblast}$ transdifferentiation in the evolution of glomerular crescent formation. Nephrol Dial Transplant 1999;14:2860-2872.

20. Orikasa $M$, Iwanaga $T$, Kawachi $H$, et al. Monoclonal antibody against rat podocyte-derived macrophagic cells reacts with crescentforming cells in an experimental model. Nephrology (Carlton) 2003;8:217-223.

21. Orikasa $M$, Iwanaga $T$, Takahashi-Iwanaga $H$, et al. Macrophagic cells outgrowth from normal rat glomerular culture: possible metaplastic change from podocytes. Lab Invest 1996;75:719-733.

22. Smeets B, Te Loeke NA, Dijkman HB, et al. The parietal epithelial cell: a key player in the pathogenesis of focal segmental glomerulosclerosis in Thy-1.1 transgenic mice. J Am Soc Nephrol 2004;15:928-939.

23. Yaoita E, Kurihara H, Sakai T, et al. Phenotypic modulation of parietal epithelial cells of Bowman's capsule in culture. Cell Tissue Res 2001;304:339-349.

24. Kalluri $R$, Cosgrove D. Assembly of type IV collagen. Insights from alpha3(IV) collagen-deficient mice. J Biol Chem 2002;75:12719-12724.

25. Cosgrove $\mathrm{D}$, Kalluri $\mathrm{R}$, Miner $\mathrm{JH}$, et al. Choosing a mouse model to study the molecular pathobiology of Alport glomerulonephritis. Kidney Int 2007;71:615-618.

26. Cosgrove D, Meehan DT, Grunkemeyer JA, et al. Collagen COL4A3 knockout: a mouse model for autosomal Alport syndrome. Genes Dev 1996;10:2981-2992.

27. Miner JH, Sanes JR. Molecular and functional defects in kidneys of mice lacking collagen alpha 3(IV): implications for Alport syndrome. J Cell Biol 1996;135:1403-1413.

28. Prodromidi El, Poulsom R, Jeffery R, et al. Bone Marrow Derived-Cells Contribute to Podocyte Regeneration and Amelioration of Renal Disease in a Mouse Model of Alport Syndrome. Stem Cells 2006;24: 2448-2455. 
29. Sugimoto $H$, Mundel TM, Sund $M$, et al. Bone-marrow-derived stem cells repair basement membrane collagen defects and reverse genetic kidney disease. Proc Natl Acad Sci USA 2006;103:7321-7326.

30. Mombaerts $P$, lacomini J, Johnson RS, et al. RAG-1-deficient mice have no mature B and T lymphocytes. Cell 1992;68:869-877.

31. Moeller MJ, Soofi A, Hartmann I, et al. Podocytes populate cellular crescents in a murine model of inflammatory glomerulonephritis. J Am Soc Nephrol 2004;15:61-67.

32. Ninichuk V, Gross $\mathrm{O}$, Reichel $\mathrm{C}$, et al. Delayed chemokine receptor 1 blockade prolongs survival in collagen 4A3-deficient mice with Alport disease. J Am Soc Nephrol 2005;16:977-985.

33. Donadelli R, Abbate M, Zanchi $C$, et al. Protein traffic activates NF-kB gene signaling and promotes MCP-1-dependent interstitial inflammation. Am J Kidney Dis 2000;36:1226-1241.
34. Harris RC, Neilson EG. Toward a unified theory of renal progression. Annu Rev Med 2006;57:365-380.

35. Shimizu $\mathrm{H}$, Maruyama $\mathrm{S}$, Yuzawa $\mathrm{Y}$, et al. Anti-monocyte chemoattractant protein-1 gene therapy attenuates renal injury induced by protein-overload proteinuria. J Am Soc Nephrol 2003;14:1496-1505.

36. Kuroiwa T, Schlimgen R, Illei GG, et al. Distinct $T$ cell/renal tubular epithelial cell interactions define differential chemokine production: implications for tubulointerstitial injury in chronic glomerulonephritides. J Immunol 2000;164:3323-3329.

37. Neilson EG. The nephritogenic T lymphocyte response in interstitial nephritis. Semin Nephrol 1993;13:496-502.

38. Strutz F, Neilson EG. The role of lymphocytes in the progression of interstitial disease. Kidney Int Suppl 1994;45:S106-S110. 